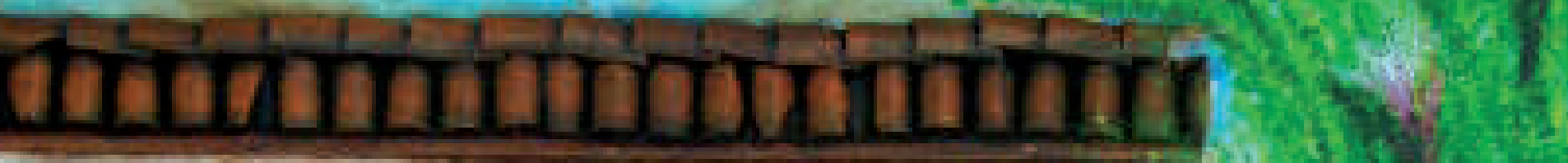

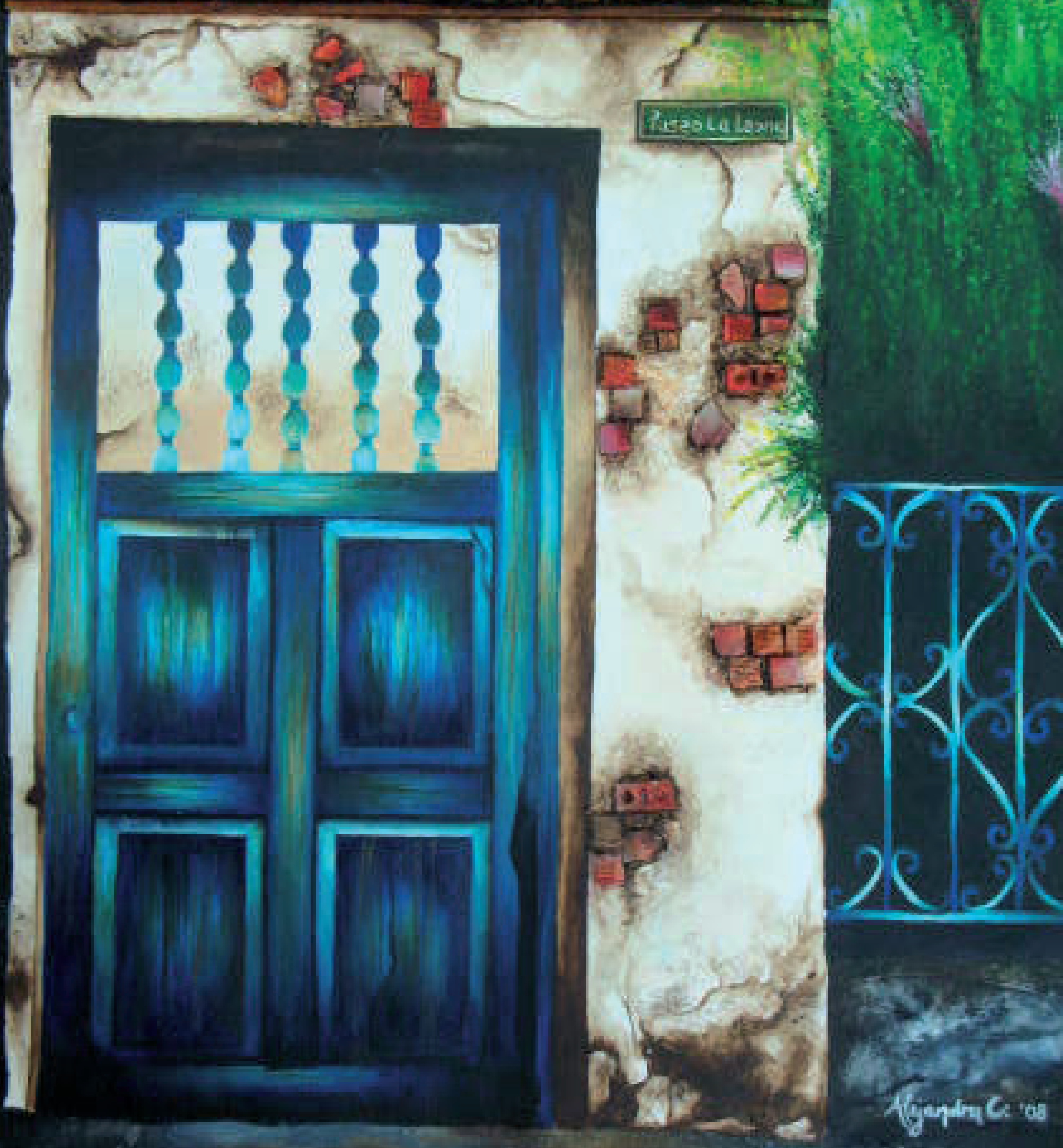




\section{El modelo de descentralización de los servicios de salud en el municipio de Langue, departamento de Valle como proyecto piloto \\ Janine Gallardo Cruz*}

RESUMEN. El modelo de gestión descentralizado de salud, implementado en el municipio de Langue, por convenio establecido entre la Secretaria de Salud y la Mancomunidad de Municipios Fronterizos "MAFRON". Es una oportunidad insuperable de mejorar la calidad de vida y la situación de salud de cada una de las comunidades beneficiadas de dicho municipio, haciendo énfasis en la atención integral materno infantil, promoviendo con ello el parto institucional; y teniendo como objetivo primordial la reducción acelerada de las muertes maternas e infantiles por causas prevenibles y que tienen mayor impacto en las poblaciones postergadas de difícil acceso a los servicios de atención primaria en salud.

El modelo permite además, en forma inmediata resolver problemas de abastecimiento de medicamentos, logística e insumos, así como la falta de recurso humano en las diferentes unidades de salud que integran la red de servicios en el municipio. A la vez este modelo permite a la comunidad participar en forma activa y social del desempeño de los empleados y el uso de los recursos económicos producidos en la unidad de salud, así como una mayor organización para contribuir en la gestión y administración de los servicios y en la conservación de la salud mediante la práctica de hábitos y estilo de vida apropiado para los pobladores del municipio.

Palabras clave: modelo, descentralización, mancomunidad.

\section{INTRODUCCIÓN}

La Organización Mundial de la Salud ha venido promoviendo la Atención Primaria de la Salud (APS), desde antes de 1978, fecha en la que se adoptó como estrategia central para alcanzar el objetivo de "Salud para Todos". Desde entonces, el mundo y la APS han cambiado drásticamente.

El propósito de la renovación de la APS es revitalizar la capacidad de todos los países de organizar una estrategia coordinada, efectiva y sostenible que permita resolver los problemas de salud existentes, afrontar los nuevos desafíos en salud y mejorar la equidad.

Se considera que una estrategia renovada de APS es una condición esencial para lograr los Objetivos de Desarrollo del Milenio a nivel internacional, como los contenidos en la Declaración del Milenio de las Naciones Unidas (ODM) así como para abordar las causas fundamentales de la salud. La nueva definición se centra en el sistema de salud en su conjunto; incluye a los sectores público, privado y sin fines de lucro, distingue valores, principios y elementos, subrayando la equidad, solidaridad e incorpora nuevos principios como la sostenibilidad y orientación hacia la calidad.

Teniendo el contexto anterior, en Honduras se está implementando un sistema de salud basado en la Atención Primaria en Salud renovada (APS-R), ya que permitirá dar respuesta al perfil epidemiológico de acumulación del país, el cual es caracterizado por una persistencia de las enfermedades transmisibles y un crecimiento rápido de las enfermedades no transmisibles, sumado a lo anterior, se encuentran lesiones de causa externa como resultado 
de la violencia (en sus diferentes tipos) ocasionado por los estilos de vida. Entre las causas de mortalidad general persisten las originadas en el período perinatal y se mantienen las muertes maternas ocasionadas por causas prevenibles durante el parto y postparto.

Con el propósito de cumplir con algunos de los $\mathrm{Ob}$ jetivos de Desarrollo del Milenio (Reducir la Mortalidad Infantil, Mejorar la Salud Materna y Combatir el VIH/ SIDA, el paludismo y otras enfermedades), la Secretaría de Salud se encuentra trabajando el componente de Fortalecimiento de las Capacidades Locales, el cual comprende un conjunto de servicios y acciones de apoyo a los gobiernos locales y otras instancias, a fin de asegurar una gestión eficiente, eficaz, sostenible y participativa para lograr un proceso de descentralización y desarrollo local exitoso.

Gracias a la capacidad técnica, administrativa de la Mancomunidad de Municipios Fronterizos (MAFRON) el municipio de Langue, Valle, como proyecto piloto del Departamento de Valle, está siendo beneficiado con el proceso de descentralización de los Servicios de Salud en todas sus comunidades; proyecto que se implementó a partir de un perfil epidemiológico cuyos resultados evidenciaron problemas en las áreas de la mortalidad materno-infantil, los factores de accesibilidad (postergación geográfica, cultural y económica) y las necesidades reales de extensión y sostenibilidad de cobertura de atención en salud continua y de calidad, justifican el esfuerzo institucional por lograr un incremento de acceso a servicios de salud de la población.

\section{OBJETIVOS DE LA INVESTIGACIÓN}

\subsection{Objetivo General}

Presentar el funcionamiento del Modelo de Descentralización de los Servicios de Salud en el municipio de Langue, departamento de Valle.

\subsection{Objetivo Específico}

- Describir el proceso de descentralización de los servicios de salud en el municipio de Langue, Valle.
- Presentar las fases del proyecto de implementación del modelo de descentralización.

- Conocer la problemática del personal de la Secretaria de Salud ante los cambios que genera el contrato de gestión y provisión de servicios de salud para la extensión de cobertura en atención primaria para el municipio de Langue.

- Identificar las instancias responsables del proceso de descentralización en el municipio de Langue, Valle.

- Identificar las acciones implementadas por las instancias responsables del proceso de descentralización de los servicios de salud en el municipio de Langue, Valle.

- Investigar las acciones que el proyecto de descentralización ha llevado a cabo en relación a la mortalidad materna infantil.

- Inventariar el talento humano directamente vinculado con el proceso de descentralización y tipo de contratación.

- Identificar las ventajas y desventajas que está generando el proyecto de descentralización de los servicios de salud implementado en el municipio de Langue.

- Hacer recomendaciones al modelo descentralizado en Langue.

\section{DIAGNÓSTICO DEL MUNICIPIO DE LANGUE}

\subsection{Datos Históricos:}

El municipio de Langue está ubicado en el departamento de Valle específicamente al norte del departamento; cuenta con 6 aldeas y 112 caseríos.

El nombre del municipio tiene su origen en la lengua de la etnia mejicano y significa "lugar de los carrizos".

La feria patronal del municipio se celebra el 13 de junio en honor a su patrono San Antonio de Padua.

\subsection{Características Geográficas}

El relieve del municipio de Langue es ligeramente accidentado, la mayoría del territorio (89\%), con una zona de poca elevación de unos $100 \mathrm{msnm}$ en las riberas del río Goascorán con pendientes leves entre 0-12\%, luego 
se eleva hacia el oeste, llegando a alcanzar elevaciones de 800 msnm con pendientes entre $12-17 \%$.

Desde Tegucigalpa hasta el casco urbano de Langue hay unos $150 \mathrm{Kms}$. En vehículo directo ese recorrido demora casi dos horas en carretera pavimentada. Desde la cabecera municipal hacia la mayoría de las aldeas se carece de ruta de buses, especialmente en la época de invierno. Las distancias a pie se recorren entre una ó más horas. El transporte de una persona enferma en vehículo directo desde una aldea hasta el casco urbano municipal puede costar más de US $\$ 25$.

Las tierras del municipio de Langue son cálidas con una precipitación anual baja de unos 1300 a $1600 \mathrm{~mm}$ al año. El patrón de lluvias irregular da como resultado un bosque seco en donde prevalecen especies forestales deciduos (hoja caduca) de hoja ancha y espinosas. En el pasado, los bosques estaban poblados de robles, guanacaste, unas pocas caobas, guapinoles, mangles y una infinidad de acacias y de árboles con espinas.

\subsection{Límites y extensión}

El municipio de Langue tiene una extensión de 135. $50 \mathrm{Km}^{2}$, lo que representa el $8.18 \%$ del territorio del departamento, colinda al norte con el municipio de Curaren (Francisco Morazán), al este con el municipio de San Francisco de Coray, al sur con el municipio de Nacaome y al oeste con el municipio de Goascorán (Ver mapa No. 1).

Parte del municipio de Langue se encuentra dentro de la cuenca del río Goascorán, y la mayor porción del territorio se ubica en la cuenca del río Nacaome. Existe una red de quebradas en todo el municipio que drenan hacia estos ríos; la mayoría de estas quebradas tienen agua en la época lluviosa pero permanecen sin agua en la época seca.

\subsection{Datos Poblacionales}

De acuerdo al último censo realizado en el 2001, la población del municipio de Langue era de 20,169 habitantes, de los cuales el $19.03 \%$ vivían en la cabecera municipal y el restante $80.97 \%$ vivían en la zona rural

\section{Mapa No. 1. Municipios del Departamento de Valle}

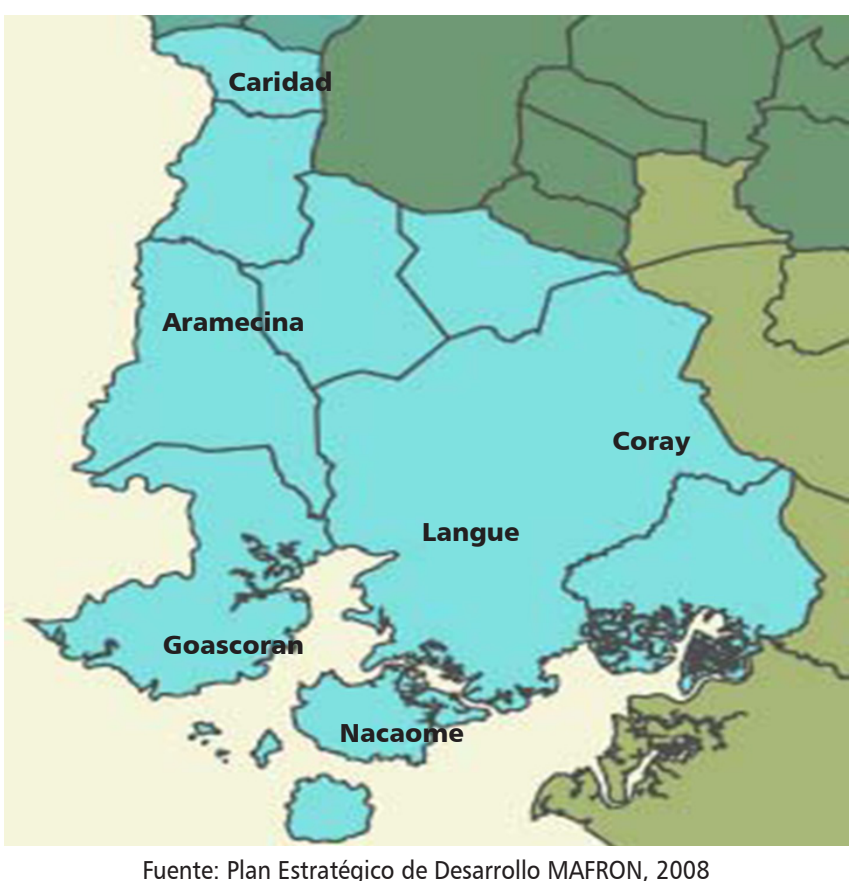

del municipio. La mayoría de la población del municipio de Langue puede considerarse joven: el 86\% es menor de 50 años, de los cuales el $46 \%$ son menores de 15 años, lo que indica una creciente demanda sobre los recursos y servicios del municipio. Las personas mayores de 50 años representan el 14\% de la población. La proporción de varones en el grupo de edad de 15-49 años es significativamente menor (ver gráfico No. 1). Esto refleja la migración de jóvenes a Estados Unidos u otros centros urbanos del país en busca de mejores oportunidades.

Las proyecciones poblacionales realizadas por el INE al 2007, permiten proyectar las distribución de la población por área de residencia, sexo y grupos de edad, en tal sentido, se sigue observando la misma tendencia del 2001 con predominio de población joven en el municipio (Ver cuadro No. 1).

\subsection{Aspectos Educativos}

El municipio de Langue, cuenta con 91 centros educativos con una población estudiantil al 2009 de 7085 estudiantes en todos los niveles (Ver cuadro No. 2).

Existen en el municipio un total de 269 maestros. 
Gráfico No. 1. Municipio de Langue: Población por Grupos de Edad

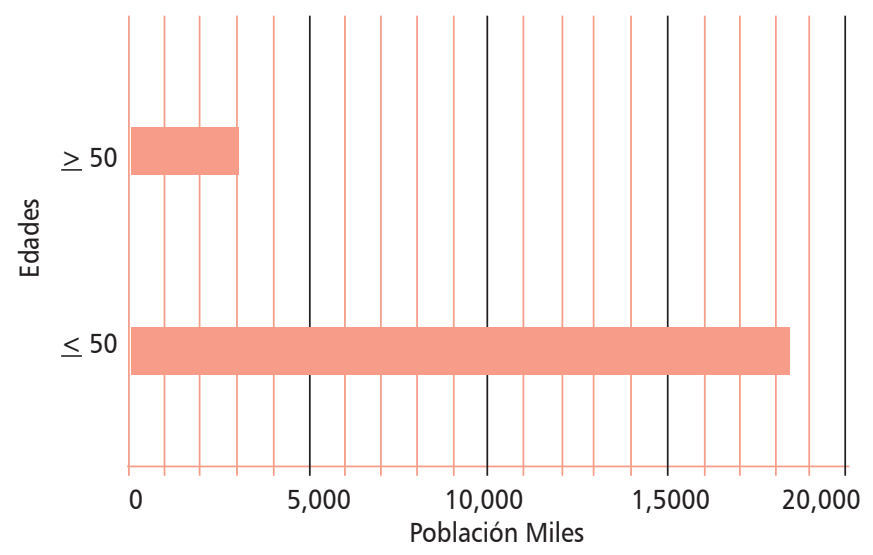

Fuente: INE. CPV 2001
Respecto a las metas planteadas por la Estrategia de Reducción de la Pobreza (ERP) para el 2006 en educación, Langue debió alcanzar las metas presentadas enel Cuadro No. 3).

Es importante apuntar que en el municipio funcionan diversos programas de educación no formal, tales como los centros PROHECO, ADEL, CCEPREB, EDUCATODOS, entre otros, éstos son manejados por voluntarios de las comunidades debidamente capacitados y tienen el respaldo de la Secretaría de Educación y de la municipalidad.

\subsection{Infraestructura y Servicios}

Debido a las condiciones normales del ecosiste-
Cuadro No. 1. Municipio de Langue: Distribución de la Población por Área de Residencia, Sexo y Grupos de Edad

\begin{tabular}{|c|c|c|c|c|c|c|c|c|}
\hline \multirow{2}{*}{$\begin{array}{c}\text { Municipio, área } \\
\text { y sexo }\end{array}$} & \multicolumn{8}{|c|}{ Grupos de Edad } \\
\hline & Total & $0 * 3$ & $4^{*} 6$ & $7 * 12$ & $13 * 17$ & $18 * 24$ & $25 * 64$ & $65+$ \\
\hline LANGUE & 21,202 & 2,347 & 1,800 & 3,443 & 2,750 & 2,799 & 6,903 & 1,160 \\
\hline Hombres & 10,612 & 1,184 & 898 & 1,796 & 1,405 & 1,498 & 3,327 & 505 \\
\hline Mujeres & 10,589 & 1,164 & 902 & 1,647 & 1,345 & 1,300 & 3,576 & 656 \\
\hline URBANO & 4,377 & 333 & 315 & 595 & 590 & 595 & 1,651 & 298 \\
\hline Hombres & 2,003 & 171 & 138 & 310 & 283 & 281 & 708 & 111 \\
\hline Mujeres & 2,374 & 162 & 176 & 285 & 307 & 314 & 943 & 187 \\
\hline RURAL & 16,825 & 2,014 & 1,485 & 2,848 & 2,160 & 2,203 & 5,252 & 863 \\
\hline Hombres & 8,609 & 1,013 & 759 & 1,486 & 1,122 & 1,218 & 2,619 & 393 \\
\hline Mujeres & 8,215 & 1,001 & 726 & 1,362 & 1,038 & 986 & 2,633 & 469 \\
\hline
\end{tabular}

Fuente: INE, 2007

\section{Cuadro No. 2. Municipio de Langue:}

Cobertura Educativa, 2009

\begin{tabular}{|l|c|c|}
\hline \multicolumn{1}{|c|}{ Nivel Escolar } & $\begin{array}{c}N^{0} \text { de centros } \\
\text { educativos }\end{array}$ & $\begin{array}{c}\text { Matrícula al } \\
2009\end{array}$ \\
\hline Nivel Preescolar & $\mathbf{4 5}$ & $\mathbf{9 1 1}$ \\
\hline Jardines formales & 19 & 588 \\
\hline CCEPREB & 22 & 267 \\
\hline Jardines PROHECO & 4 & 56 \\
\hline Nivel Primaria Básica & 44 & 4429 \\
\hline Escuelas Formales & 29 & 2502 \\
\hline Escuelas PROHECO & 5 & 228 \\
\hline Escuela de adultos & 1 & 20 \\
\hline C.E.B. (1ro. A 6to.) & 9 & 1135 \\
\hline C.E.B. (7mo. A 9no.) & 2 & 544 \\
\hline Nivel Media & 1 & 1654 \\
\hline Media Presencial & 1 & 1492 \\
\hline ISEMED (Educación media a & & 162 \\
\hline distancia) & Fuente: Dirección Distrital del Municipio de Langue, Valle, 2009 \\
\hline
\end{tabular}

ma seco-árido y la estacionalidad de las lluvias, esto condiciona la disponibilidad y acceso al agua para el consumo humano, creando problemas para la población y su salud, para la municipalidad la solución de éste problema se ha convertido en una actividad prioritaria y una necesidad indispensable para satisfacer a la población.

En relación a la dotación de agua, la situación del municipio es bastante inferior a la media del departamento $(74.06 \%)$, y en la disposición de excretas es muy baja con respecto a la media del departamento (81.77\%).

El municipio de Langue cuenta con 3,300 viviendas de éstas 1,442 reciben agua del sector público; 600 viviendas la reciben de pozos de malacates; 213 la reciben de pozos con bombas; 975 reciben agua de vertientes o ríos, 13 la reciben de lagos o lagunas; 4 la reciben de vendedores privados o repartidores y 53 viviendas la reciben de cualquier otra forma (Ver cuadro No. 4).

Respecto a la red sanitaria y específicamente la disposición de excretas en el 2001 se registró que un 
Cuadro No. 3. Plan de Metas a alcanzar al 2009

\begin{tabular}{|c|l|c|c|}
\hline$\#$ & \multicolumn{1}{|c|}{ Meta } & $\%$ a alcanzar & \% alcanzado \\
\hline 1 & Graduados del sexto grado & $82 \%$ & Logrado \\
\hline 2 & $\begin{array}{l}\text { Graduación de sexto grado } \\
\text { con 12 años }\end{array}$ & $35 \%$ & Logrado \\
\hline 3 & $\begin{array}{l}\text { Matricula de niños de 7 años } \\
\text { en 1er. grado }\end{array}$ & $78 \%$ & Logrado \\
\hline 4 & $\begin{array}{l}\text { Matricula de niños menores } \\
\text { de 7 años en 1er. grado }\end{array}$ & $78 \%$ & Logrado \\
\hline 5 & $\begin{array}{l}\text { Rendimiento académico en } \\
\text { español y matemáticas }\end{array}$ & $55 \%$ & Logrado \\
\hline 6 & Deserción Escolar & $1.1 \%$ & Superado \\
\hline 7 & Reprobación de 1er. grado & $5.0 \%$ & $0.00 \%$ \\
\hline 8 & Reprobación de 2do. grado & $3.1 \%$ & $0.00 \%$ \\
\hline 9 & Reprobación de 3er. grado & $3.0 \%$ & $0.00 \%$ \\
\hline 10 & Reprobación de 4to. grado & $2.0 \%$ & $0.00 \%$ \\
\hline 11 & Reprobación de 5to. grado & $1.2 \%$ & $0.00 \%$ \\
\hline 12 & Reprobación de 6to. grado & $0.3 \%$ & $0.00 \%$ \\
\hline
\end{tabular}

Fuente: ERP, 2006

\section{Cuadro No. 4. Municipio de Langue:} Procedencia del Agua

\begin{tabular}{|l|c|c|}
\hline \multicolumn{1}{|c|}{ Categorías } & Casos & $\%$ \\
\hline 1. De tubería del sistema público/privado & 1442 & 43.7 \\
\hline 2. De pozo malacate & 600 & 18.2 \\
\hline 3. De pozo con bomba & 213 & 6.5 \\
\hline 4. De vertiente, rio & 975 & 29.5 \\
\hline 5. De lago o laguna & 13 & 0.4 \\
\hline 6. De vendedor o repartidor & 4 & 0.1 \\
\hline 7. Otro Total & 53 & 1.6 \\
\hline
\end{tabular}

Fuente: Elaboración propia en base a los datos del XVI Censo de Población y Vivienda, INE 2001

$55.5 \%$ de la población carecía de algún tipo de servicio sanitario por lo que en el 2006 la ERP planteó en el municipio de Langue la meta de incrementar la cobertura de este servicio logrando que el $52 \%$ de la población cuente con algún método de disposición de excretas (Ver cuadro No. 5).

El municipio no cuenta con un sistema de tren de aseo ni rellenos sanitarios, por lo que el manejo de la basura es un problema ambiental importante. Tradicionalmente los pobladores han transportado la basura a una serie de botaderos dispersos, en la orilla de las carreteras, en gran parte de los casos (77.1\%) la queman o entierran.

\section{Cuadro No. 5. Municipio de Langue:}

Tipo de Servicio Sanitario

\begin{tabular}{|l|c|c|}
\hline \multicolumn{1}{|c|}{ Categorías } & Casos & $\%$ \\
\hline 1. Inodoro red alcantarillado & 13 & 0.4 \\
\hline 2. Inodoro pozo séptico & 163 & 4.8 \\
\hline 3. Inodoro con descarga a rio & 3 & 0.1 \\
\hline 4. Letrina de pozo simple & 1317 & 39.1 \\
\hline 5. No tiene & 1869 & 55.5 \\
\hline Total & 3365 & 100.0 \\
\hline
\end{tabular}

Fuente: Elaboración propia en base a los datos del XVI Censo de Población y Vivienda, INE 2001.

La red vial en el municipio de Langue es bastante amplia. La principal vía de comunicación es la carretera pavimentada, que une la cabecera municipal con la carretera Panamericana. Existen carreteras intermunicipales que en época de verano comunican a los municipios de Aramecina, Nacaome, Coray y Goascorán y al Departamento de Francisco Morazán. Existe un sistema local de transporte dinámico permanente internamente en el municipio hacia lugares del departamento y del país.

\section{LOS SERVICIOS DE SALUD EN EL MUNICIPIO DE LANGUE, VALLE}

Los servicios de salud en el municipio de Langue son prestados por la Secretaria de Salud a través de la Mancomunidad de Municipios Fronterizos MAFRON ya que el grado de pobreza, perfil epidemiológico analizado y las necesidades reales de extensión de cobertura en el Municipio de Langue justifican el esfuerzo institucional por lograr un incremento de acceso a servicios de salud, atención de partos institucionales y emergencias, focalizando las intervenciones en el binomio madre-hijo.

\subsection{Condiciones Físicas de las Unidades de Salud}

Ninguna de las Unidades de Salud (USs) cuenta con licenciamiento ni cumple con las condiciones que se requiere en este aspecto. Cada unidad prestadora de servicios del municipio de Langue cuentan con su flujo de señalización y con la rotulación de espacios físicos.

Es de hacer notar que el 30\% de las Unidades de Salud (USs) cuentan con incineradores para aplicar 
reglas de bioseguridad. El aseo de los centros de salud de Langue se hace con personal voluntario, coordinados por el personal de salud.

En este municipio se cuenta con 16 unidades de salud de las cuales 3 de ellas son Cesamos y 12 son Cesares, también se cuenta con un laboratorio y un centro de odontología (Ver mapa No. 2).

En relación al personal se cuenta con 4 médicos generales, 2 licenciadas de enfermería, 18 enfermeras auxiliares, 7 promotores sociales T/A, 1 administrador, 1 odontólogo, 1 técnico de laboratorio, 1 secretaria (Mafron, 2008).

En las Unidades de Salud del municipio de Langue, Valle se brinda la atención de diversas necesidades persistentes en este municipio, a la vez podemos mencionar algunas de ellas como ser:

- Captación y detección de sintomáticos respiratorios (sintomático respiratorio, baciloscopia)

\section{Mapa No. 2. Municipio de Langue: Ubicación de las Unidades de Salud}

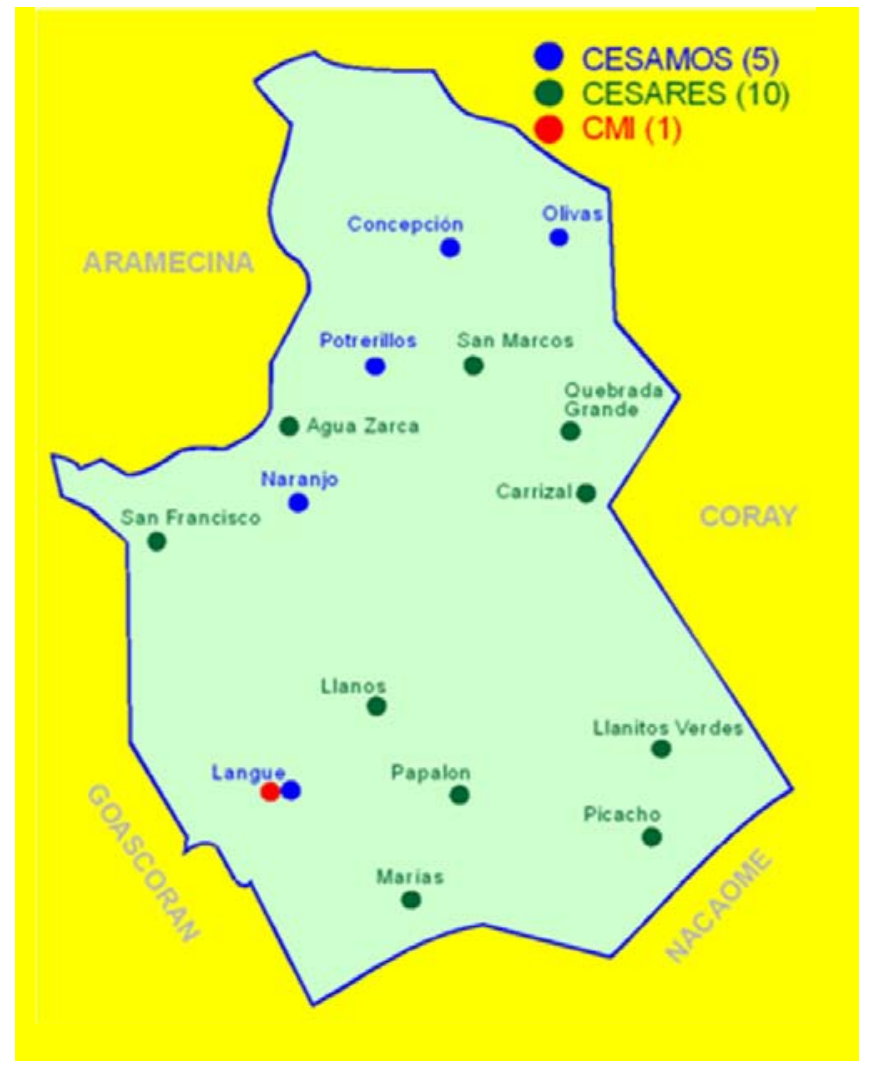

Fuente: MAFRON 2008
Cuadro No. 6. Municipio de Langue:

Eliminación de la Basura

\begin{tabular}{|l|c|c|}
\hline Categorías & Casos & \\
\hline 1. La tira a la calle & 617 & 18.7 \\
\hline 2. La recoge el carro de la basura & 12 & 0.4 \\
\hline 3. La lleva al depósito & 19 & 0.6 \\
\hline 4. La quema o entierra & 2544 & 77.1 \\
\hline 5. Paga a particulares & 80 & 2.4 \\
\hline 6. Otro & 28 & 0.8 \\
\hline Total & 3300 & 100.0 \\
\hline
\end{tabular}

Fuente: Elaboración propia en base a los datos del XVI Censo de Población y Vivienda, INE 2001.

- Atención integral a la mujer (Planificación Familiar)

- Atenciones de salud mental (epilepsia, episodio depresivo, agresión por fuerza corporal trastornos neuróticos).

- Captación y seguimientos a embarazos nuevos (riesgos reproductivos, etc.).

- Vacunaciones (Polio, Pentavalente, DPT, SRP, BCG, Toxoide Tetánico y Diftérico, Hepatitis B, Influenza, Vitamina "A", TD).

- Captación y seguimiento a los pacientes con ITS/ SIDA.

- Captación y seguimiento de nuevas atenciones por morbilidad (Varicela, Diarrea, Disentería, Asma y Bronquitis, Neumonía Bronconeumonia, Faringo Estreptococo, Otras Faringoamigdalitis).

El cuadro No. 7 presenta la nuevas captaciones y atención por síntomas generales en cada Unidad de Salud en el municipio de Langue, Valle.

\section{PROCESO DE DESCENTRALIZACION DE LOS SER- VICIOS DE SALUD EN EL MUNICIPIO DE LANGUE, VALLE}

El modelo Descentralizado de Atención de Salud llega en al municipio de Langue al inicio como una oportunidad complementaria de cubrir necesidades indispensables con el propósito de mejorar la calidad de los servicios de salud a la población de Langue. 
Cuadro No. 7. Región Departamental de Salud No. 17: Atenciones por Grupos Etáreos por USS y municipio. Primer Año del Proyecto. Periodo Agosto

\begin{tabular}{|c|c|c|c|c|c|c|}
\hline \multirow{3}{*}{ U S / Municipios } & \multirow{3}{*}{$\begin{array}{l}\text { Población } \\
\text { General }\end{array}$} & \multicolumn{5}{|c|}{ Atención Generales } \\
\hline & & \multirow{2}{*}{ Nvas } & \multirow{2}{*}{$\%$} & \multirow{2}{*}{ S-Sig } & \multirow{2}{*}{ C } & Total Atenc. \\
\hline & & & & & & $(\mathrm{N}+\mathrm{S})$ \\
\hline Langue & 6,098 & 128 & $2.1 \%$ & 489 & 5 & 617 \\
\hline Olivas & 1,180 & 0 & $0.0 \%$ & 164 & 0 & 164 \\
\hline San Marcos & 741 & 64 & $8.6 \%$ & 171 & 4 & 235 \\
\hline Concepción de Maria & 1,576 & 37 & $2.3 \%$ & 127 & 4 & 164 \\
\hline Potrerillos & 869 & 3 & $0.3 \%$ & 142 & 48 & 145 \\
\hline Naranjo & 1,515 & 80 & $5.3 \%$ & 430 & 6 & 510 \\
\hline Agua Zarca & 609 & 26 & $4.3 \%$ & 81 & 4 & 107 \\
\hline Los llanos & 920 & 32 & $3.5 \%$ & 72 & 3 & 104 \\
\hline Carrizal & 2,278 & 0 & $0.0 \%$ & 114 & 0 & 114 \\
\hline Quebrada grande & 676 & 56 & $8.3 \%$ & 75 & 2 & 131 \\
\hline Papalon & 669 & 1 & $0.1 \%$ & 118 & 119 & 119 \\
\hline Llanitos verdes & 707 & 23 & $3.3 \%$ & 38 & 3 & 61 \\
\hline Picacho & 1,229 & 0 & $0.0 \%$ & 161 & 0 & 161 \\
\hline Las Marías & 1,342 & 20 & $1.5 \%$ & 211 & 12 & 231 \\
\hline San Francisco & 802 & 23 & $2.9 \%$ & 156 & 8 & 179 \\
\hline Total & 21,211 & 493 & $2.3 \%$ & 2,549 & 6 & 3,042 \\
\hline
\end{tabular}

Fuente: Césamo, Langue

Los servicios están dirigidos a áreas como ser insumos (medicamentos, papelería, capacitaciones), logística (combustible, viáticos, mantenimiento de motocicletas y dotación de las mismas) y contratación de recurso humano en las diferentes unidades de salud que conforman la red de atención en salud en el municipio; en vista de que la Secretaría de Salud representada por la Región Departamental de Salud de Valle por problemas presupuestarios no contaba con los medios necesarios para brindar la cobertura adecuada a la demanda real existente en los aspectos antes mencionados.

En primera instancia se planteó la necesidad de construir una clínica materno infantil dotándola de equipo y la contratación del recurso humano necesario para su funcionamiento, asegurando una atención de calidad y eficiencia.

Además se evidenció la necesidad de complementar las plazas de enfermeras auxiliares, promotores y médicos en lugares estratégicos de las unidades de salud que estaban siendo atendidos por personal de contrato y de servicio social. También fue evidente la necesidad de insumos y logística así como de equipo médico para que la población pudiera recibir una atención de calidad y de forma oportuna que les permitiera mejorar su calidez y calidad de vida reduciendo la incidencia de muertes y enfermedades sobre todo maternas e infantiles en consonancia con dos de los objetivos y metas del milenio.

\section{FASES DEL PROYECTO}

El proceso de descentralización en el municipio de Valle se desarrolló en 3 fases desglosadas de la siguiente manera:

En la primera fase del proyecto se realizó un diagnóstico del espacio poblacional del municipio de Langue en el que se presentaron las características siguientes: El contexto socio demográfico del municipio, el perfil epidemiológico, la situación actual de la red de servicios de salud en el municipio y las razones de elegibilidad del municipio.

En la segunda fase se comienza a elaborar el diseño de los modelos a implementar en el municipio; dentro esos modelos se encontraron 3:

- El Modelo de Atención: éste modelo cuenta con una cartera de servicios posibles, la organización de la provisión, los niveles de atención a los pacientes y el recurso humano necesario por nivel de atención en cada US.

- El Modelo de Gestión: éste contempla el mecanismo de relación entre los involucrados en el proceso, el organigrama del proyecto a implementar, los incentivos que se brindarían, la contratación de proveedores, los procesos y mecanismos administrativos sujetos a auditorias, la evaluación de desempeño, y los términos de rescisión de convenio al no cumplirse con las expectativas del proyecto.

- El Modelo de Financiamiento: en éste modelo se contemplan los mecanismos de pago o desembolsos 
al administrador del proyecto, las garantías de calidad y las fuentes adicionales con las que estará sujeto a negociar el administrador.

El modelo de financiamiento y asignación de recursos propuestos para la gestión en Langue, se basa en los siguientes principios:

- Promueve la garantía de equidad en el acceso a los servicios de salud.

- Incentiva la calidad de los servicios y la satisfacción de los usuarios.

- Garantiza niveles de eficiencia elevados.

- Promueve una asignación de recursos costo-efectiva.

- Garantiza transparencia desde el punto de vista de la aplicación de criterios y ritmos bien definidos.

- Permite la medición de resultados y el seguimiento y evaluación del destino final de los fondos.

- Permite trabajar bajo un esquema de rendición de cuentas hacia lo interno de la institución y hacia lo externo, con la participación de la comunidad en el control del uso de los recursos.

- Incentiva la participación de las diferentes dependencias y niveles de toma de decisiones, dentro de la Secretaría de Salud, en los diferentes ámbitos, tales como planificación y control de gestión a nivel central y regional.

- Promueve el desarrollo de un modelo de atención integral en salud, debidamente articulado y coordinado entre los distintos niveles de atención.

En la tercera fase se presentan los análisis de costos respectivos correspondientes a la propuesta del proyecto como ser el presupuesto gestionado, negociado y asignado.

\section{INSTANCIAS RESPONSABLES DEL PROCESO DE} DESCENTRALIZACION

- Secretaría de Salud en el marco de la reforma y con financiamiento del Banco Mundial (USAID) un proyecto de extensión de cobertura y mejoramiento de la calidad de los servicios a través de nuevos modelos descentralizados de gestión y provisión.

- Apoyo técnico brindado por el PRSS (Encargados del seguimiento a las actividades del Convenio entre la MAFRON y la Secretaría de Salud)

- Región departamental de salud (Institución responsable de las monitorias y evaluaciones de las actividades de salud realizadas en el Municipio)

- Mancomunidad de Municipios Fronterizos MAFRON (Administrador de los Fondos asignados por la Secretaría de Salud)

- Alcaldía Municipal de Langue (Institución encargada de acompañar y verificar todo el proceso de descentralización).

\section{TALENTO HUMANO VINCULADO Y NECESARIO EN EL PROCESO DE DESCENTRALIZACION}

En el municipio de Langue el talento humano involucrado directamente en el proceso de descentralización asciende a 39 personas, tal como lo muestra el cuadro No. 8.

\section{VENTAJAS Y DESVENTAJAS DEL PROYECTO DE DESCENTRALIZACION}

\subsection{Ventajas}

- La mejora gradual de los aspectos vinculados no sólo con la producción de servicios sino también con la

\section{Cuadro No. 8. Municipio de Langue: Talento Humano involucrado en el proceso de Descentralización de los Servicios de Salud}

\begin{tabular}{|c|c|c|}
\hline Cantidad & Nombre del Cargo & Formacion Académica \\
\hline 4 & $\begin{array}{c}\text { Médicos permanentes } / \\
\text { Trabajo social }\end{array}$ & Medico o Cirujano \\
\hline 19 & $\begin{array}{c}\text { Enfermeras Auxiliares } \\
\text { Supervisoras de la Red } \\
\text { Municipal }\end{array}$ & $\begin{array}{c}\text { Auxiliar de enfermería } \\
\text { Licenciada en enfermería }\end{array}$ \\
\hline 10 & $\begin{array}{c}\text { Promotores Sociales } \\
\text { Educación Media (Promotores } \\
\text { Sociales, Maestros, Bachilleres, } \\
\text { Peritos etc.) }\end{array}$ \\
\hline 1 & Administrador & $\begin{array}{c}\text { Administrador de empresas o } \\
\text { carreras afines. }\end{array}$ \\
\hline 1 & Secretaría & Secretaría Profesional \\
\hline 1 & Odontólogo & Doctor en Odontología \\
\hline 1 & Técnico de Laboratorio & Técnico de laboratorio clínico \\
\hline
\end{tabular}

FUENTE: Convenio del proyecto de extensión de cobertura y mejoramiento de la calidad de los servicios a través de nuevos modelos descentralizados de gestión y provisión entre MAFRON y la Secretaria de Salud. 
mejora continua de la calidad en la prestación de servicios de salud.

- La extensión efectiva de la cobertura de servicios de salud para todas las comunidades del municipio de Langue.

- Una mayor ampliación de la oferta de servicios de salud mediante una gestión descentralizada de los recursos técnicos y económicos.

- El funcionamiento de los diferentes niveles de la red, mejorando su capacidad resolutiva y haciendo efectivo el sistema de Referencia de las USs.

- Beneficios a la mujer parturienta por partos institucionales.

- Gestión de mayores recursos económicos por medio de la Mancomunidad.

- Organización e involucramiento de las comunidades en la toma de decisiones en las USs.

\subsection{Desventajas}

- La mayor parte del recurso humano ya está contratado de manera permanente en el municipio.

- Presupuesto asignado.

- Recurso humano inutilizado en cuanto a las áreas de cobertura de cada una USs.

- Clínica materna no entregada e inaugurada.

- Descentralización de toma de decisiones.

\section{ACCIONES IMPLEMENTADAS POR EL PROYECTO DE DESCENTRALIZACIÓN}

10.1 Acciones implementadas por las instancias responsables del proceso de descentralización de los servicios de salud en el municipio de Langue.

El modelo de provisión de servicios de primer nivel (Promoción, Prevención y atención de la enfermedad) más la atención de partos institucionales, emergencias, referencias y acciones de salud pública para pobladores del municipio de Langue y todas sus comunidades, se basa en la implementación de:

- Un modelo descentralizado que garantice la eficiencia en el uso de los recursos, eficacia en las interven- ciones priorizadas y equidad en el financiamiento y acceso a los servicios, en el entendido de que se induce una focalización del subsidio estatal hacia la población sin capacidad de pago.

- Un contrato o convenio que contempla las actividades y responsabilidades de ambas partes (Gestor: un ente sin fines de lucro; y la Secretaría de Salud: rector financiador).

- Un mecanismo de financiamiento innovador, que permita una gestión y prestación de servicios más dinámica y adecuada a las necesidades de salud de la población.

El municipio no contaba con el licenciamiento de ninguna Unidad de Salud. En la actualidad las 15 unidades de salud que se encuentran en el municipio cuentan con su debido licenciamiento específico.

El modelo está pretendiendo mejorar gradualmente, los aspectos vinculados no sólo con la producción de servicios sino también con la mejora continua de la calidad en la prestación de los mismos a través del cumplimiento de las normas y estándares establecidos por la Secretaría de Salud, con los recursos necesarios y al menor costo.

A la vez implica extender efectivamente la cobertura de servicios de salud para todas las comunidades del municipio de Langue y ampliar la oferta de los mismos mediante una gestión descentralizadas de los recursos.

Gracias al Modelo de Atención en Salud FamiliarComunitario que se contempla en proyecto se ha logrado trabajar de manera efectiva los siguientes aspectos:

- Orientación hacia las necesidades de salud de la población basada en la promoción y prevención, pero con respuesta oportuna y efectiva en curación y rehabilitación, contribuyendo al logro de un ambiente saludable con la participación activa de la comunidad.

- Delimitación del espacio población/territorio beneficiario. Constituido por el ámbito municipal de Langue con una población estimada al 2007 de 21,202 habitantes para la atención integral de la totalidad de sus habitantes; así como la atención limitada a partos y emergencias en la CMI del casco urbano municipal. 
- Aseguramiento de una cartera de servicios de atención primer nivel orientada a la promoción, prevención, atención del enfermo, atención de partos institucionales, emergencias, referencias y acciones de salud pública, con énfasis en la atención del grupo materno infantil.

10.2 Acciones implementadas por el proceso de descentralización de los servicios de salud en relación a la mortalidad materna e infantil.

El perfil epidemiológico analizado, los factores de accesibilidad y las necesidades reales de extensión y sostenibilidad de cobertura de atención médica continua con calidad justifican el esfuerzo institucional por lograr un incremento de acceso a servicios continuos de salud y atención de partos institucionales, centrando y focalizando las intervenciones en el binomio madre-hijo.

En el gráfico No. 3 se observa que la tasa de mortalidad infantil el departamento de Valle ocupa un 32.2\% de muertes infantiles en el país, el municipio de Langue presenta el $22.7 \%$ de muertes infantiles lo que significa que es el que registra el menor porcentaje de muertes infantiles en el departamento de Valle.

Resulta necesario asegurar una oferta continuada de atención mediante unas prestaciones y un equipo de salud, que resulte adecuada al volumen y necesidades de salud de la población del municipio de Langue.

La estrategia de Enfoque de Riesgo Reproductivo y el desarrollo de procesos en la comunidad como fortalecimiento a las acciones de promoción del parto institucional que se generan en los centros de salud, requieren del funcionamiento adecuado de la clínica de atención de parto y de emergencias definidas como instancias intermedias entre la comunidad y el hospital para la atención del parto, las cuales tienen el objetivo de incrementar el parto institucional con énfasis en las mujeres que tienen factores de riesgo obstétrico y embarazos no complicados.

El municipio de Langue carecía de una Clínica Materno Infantil "CMI" y en la actualidad se encuentra en su etapa final de construcción con fondos del FHIS y gracias al proyecto será habilitada y equipada lo más pronto posible ya que los pocos partos institucionales requieren desplazamientos a otros municipios.

En este municipio no se dispone de transporte apropiado para traslado de emergencias al segundo nivel de atención como ser la Clínica Materno Infantil de Nacaome o el Hospital de San Lorenzo, Valle. En la actualidad se cuenta con una ambulancia para la clínica materno de Langue esta será utilizada para las emergencias necesarias del municipio.

\section{Gráfico No. 3. Departamento de Valle: Tasa de Mortalidad Infantil por Municipio, 2001}

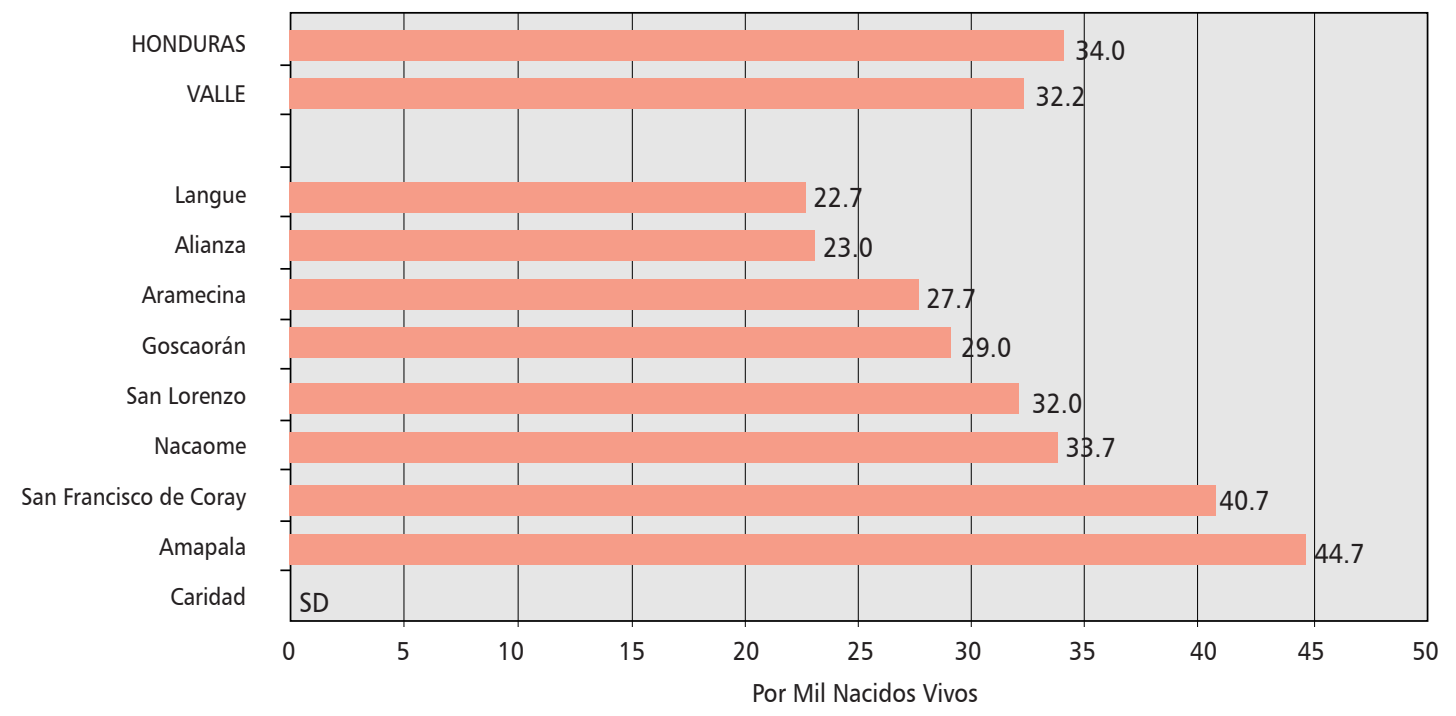

Fuente: INE. CPV 2001 
Una de las prioridades del Proceso de Descentralización de los Servicios de Salud es la captación a tiempo de las mujeres embarazadas en este municipio, ya que el perfil epidemiológico representa un alto porcentaje en la atención de partos comunitarios. Por lo que el proyecto esta brindándole a cada mujer embarazada un bono materno con el que podrá movilizarse a la hora del parto al centro hospitalario más cercano.

En el municipio de Langue las enfermedades prevenibles más comunes que se presentan en la mayor parte de las comunidades son los casos de sarampión, tosferina, el tétano, el tétano neonatal, la rubiola, el spc, la meningitis hib, la meningitis $T B$, y la hepatitis $B$, gracias al proyecto se mantienen abastecidas la mayor parte de las Unidades de Salud según el cuadro básico de medicamentos.

También se ha logrado organizar en las comunidades Comités de Salud con el objetivo de una mayor captación de embarazos, apoyo comunitario a los centros de salud, fortalecimiento de capacidades en las comunidades con capacitaciones, intercambios de experiencias y apoyo en la gestión de recursos tanto económicos como materiales para el mejoramiento de los centros de salud.

\section{RECOMENDACIONES}

- Antes, durante y con la implementación de los Procesos de Descentralización en la Secretaria de Salud se pretende mejorar la calidad y los servicios de salud en las comunidades del municipio de Langue pero para ello es necesario fortalecer los gobiernos locales (alcaldías, recurso humano contratado, sociedad civil, etc.) para que los mismos brinden el apoyo necesario al proyecto.

- En cuanto a la Secretaría de Salud es recomendable que los presupuestos que se le asignen a los municipios en donde se estén ejecutando procesos de descentralización de salud sean presupuestos acorde a la realidad y situación del municipio beneficiado.

- Las Direcciones Regionales de Salud son entes gubernamentales aliados y necesarios para el éxito de los procesos de descentralización de los servicios de salud. A la vez es oportuno expresar que de parte de los mismos, debe existir solamente un acompañamiento en la toma de decisiones, esto permitiría un mayor involucramiento y apropiamiento del proyecto por la institución administradora.

- La Mancomunidad MAFRON como ente administrador y responsable del funcionamiento y puesta en marcha del proyecto de Descentralización de los servicios de salud en el municipio de Langue deberá apropiarse más del mismo. Ya que dicho proyecto es piloto para los otros municipios que conforman la Mancomunidad MAFRON.

\section{CONCLUSIONES}

$>$ El momento histórico político vivido en el país incidió e influyó en el no cumplimiento de los objetivos esperados por parte del proyecto.

$>$ Se percibe una incertidumbre de parte de las autoridades centrales y locales en cuanto al apoyo técnico y financiero que permita la consolidación de los procesos de descentralización implementados.

Pese a los inconvenientes administrativos y financieros que han surgido en el proyecto las metas y objetivos establecidos se han logrado; sin embargo, las autoridades locales perciben en el tiempo alguna amenaza que interfiera en la continuidad del proyecto debido a la claridad en las políticas públicas del país.

\section{BIBLIOGRAFÍA}

- Instituto Nacional de Estadística, Censo Poblacional de Viviendas 2001.

- Instituto Nacional de Estadística. Proyecciones de Población 2007.

- $\quad$ Plan Estratégico de Desarrollo Municipal de Langue, Valle, 2008

- Plan Estratégico de Desarrollo de la Mancomunidad MAFRON, 2008.

- $\quad$ Centro de Salud de Langue, Valle, 2009.

- $\quad$ Dirección Distrital de Langue, Valle, 2009.

- Región Sanitaria Departamental de Valle, 2009. 